\title{
Ganglioneuroblastoma with Extensive Vascular Encasement: A Case Report
}

\author{
Juneja $A^{1}$, Thakral A ${ }^{1}$, Thakral D A , Hiralal $^{1}$ \\ ${ }^{1}$ Department of Radiodiagnosis, ${ }^{3}$ Department of Laboratory Hematology, Sanjay Gandhi Post \\ Graduate Institute of Medical Sciences, Lucknow, India
}

\begin{abstract}
Ganglioneuroblastoma is a tumor of the sympathetic nervous system in paediatric age group that is intermediate in behavior between ganglioneuroma and neuroblastoma. We present a case of a 3 year old girl who presented with a large long standing abdominal mass. Ultrasonographic features were suggestive of large retroperitoneal mass with calcific foci \& extensive vascular encasement consistent with neurogenic tumor. Contrast enhanced computed tomography (CT) performed for staging confirmed these findings while histopathologic diagnosis of ganglioneuroblastoma was achieved by ultrasound guided biopsy. While vascular encasement is known in ganglioneuroblastoma, the scale of vascular encasement in this case is extensive.
\end{abstract}

Keywords: Computed Tomography, Ganglioneuroblastoma, Neuroblastoma, Ultrasonography

\section{Introduction}

Ganglioneuroblastoma is a tumor of the sympathetic nervous system that may be seen in the adrenal gland, retroperitoneum and other sites. It is intermediate in behaviour between benign ganglioneuroma (slow growing and unlikely to spread) and malignant neuroblastoma (fast growing, aggressive, and likely to spread). It usually occurs in young children with equal frequency in either $\operatorname{sex}^{1}$ presenting as abdominal mass.

Correspondence to: Dr. Hiralal, Associate Professor, Department of Radiodiagnosis, Sanjay Gandhi Post Graduate Institute of Medical Sciences, Rai Barielly Road, Lucknow, Uttar Pradesh, Pin-226014

E-mail: hiralal2007@yahoo.co.in

\section{Case Report}

A 3 year old girl presented with complaints of abdominal fullness since the age of 8 months. On examination, a large lobulated mass was felt in the left hypochondrium and lumbar region which was firm in consistency. Laboratory examination revealed normal blood counts and hormonal levels.

Abdominal Ultrasonography revealed a large heteroechoic retroperitoneal mass lesion measuring approximately $11 \times 8 \mathrm{~cm}$ causing displacement of bowel loops to the right side, displacement of pancreas and right kidney anteriorly. The mass encasing celiac, superior and inferior mesenteric \& left renal vessels. There were small echogenic foci 
causing posterior acoustic shadowing suggestive of calcification.

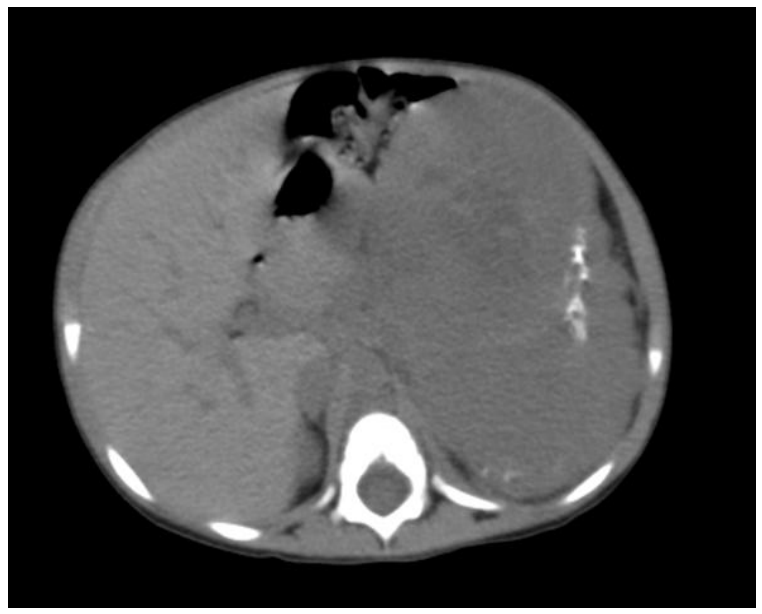

Fig 1: Plain CT abdomen showing a large mass lesion involving the left hemi abdomen and showing specks of stippled calcification.

Based on ultrasonographic findings, contrast enhanced triple phase CT scan (Fig 1\&2) was performed for localisation \& characterisation of the mass, revealing a large lobulated mass lesion measuring approximately $12 \times 10 \times 7 \mathrm{~cm}$. It showed soft tissue density (10 - 38 HU on unenhanced scans) with post contrast enhancement (40 $156 \mathrm{HU})$ in the solid regions. Discrete foci of calcification were seen within the mass lesion. Encasement of celiac artery and its branches (splenic and common hepatic artery), left renal, superior and inferior mesenteric arteries and veins was seen without compromising their lumen. Pancreas, stomach and small bowel loops were displaced anteriorly and to the left by the mass.

The mass was and abutting and displacing the kidney anteriorly with stretching of the main renal artery and vein. Bilateral adrenal glands could not be identified separately.
Liver and spleen were normal. Bone window did not reveal any metastatic focus.

Based on CT findings, the differential diagnosis of malignant neurogenic tumor or a conglomerate lymph nodal mass was made.

USG guided biopsy was performed from the mass lesion. Histopathology sections (Fig 3) showed tumor composed of two cell populations, predominantly ganglion cells which were large polygonal with abundant eosinophilic cytoplasm, round vesicular eccentric nuclei and prominent nucleoli in a background of spindled schwannian stroma. Few binucleated ganglion cells were also seen. Intermixed between them were small clusters of small cells with scant cytoplasm, hyperchromatic nuclei and inconspicuous nucleoli in a fine fibrillary neuropil like stroma. Immunohistochemical stain- shows positivity of tumor cells for chromogranin.

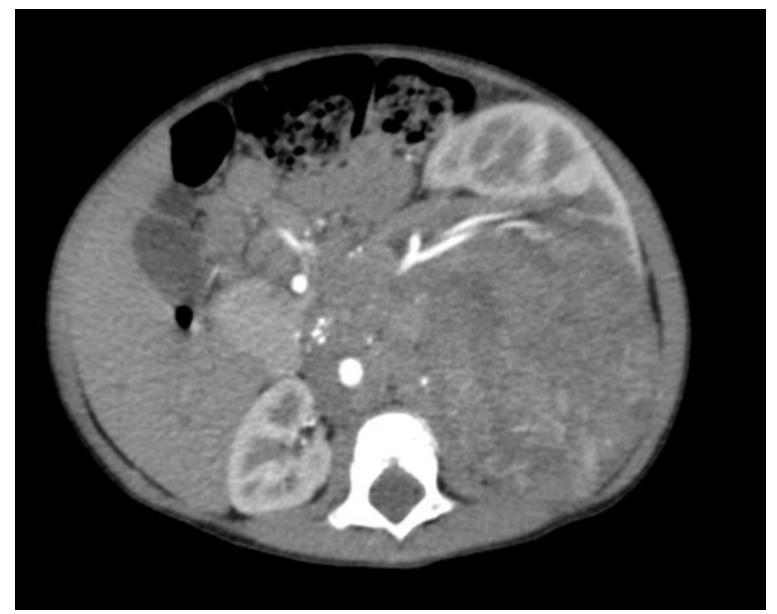

Fig 2: Arterial phase CT showing a large mass with heterogeneous arterial enhancement \& extensive encasement of aorta $\&$ left renal vessels.

MIBG whole body scan was performed at 24 and $72 \mathrm{hrs}$ after administration of 500 micro curie of I-131 MIBG revealing a large focus of abnormal tracer uptake in left side of the abdomen. Rest of the body revealed 
physiological tracer uptake with no scan evidence of any metastasis.

\section{Discussion}

The term 'ganglioneuroblastoma' was first applied by Robertson in 1915 to neurogenic tumor with combined histologic features of ganglioneuroma and neuroblastoma. Ganglioneuroblastoma is composed of both mature gangliocytes and immature neuroblasts and has intermediate malignant potential. The most common sites of origin of neuroblastoma and ganglioneuroblastoma, in order, are the adrenal medulla (35\%), extraadrenal retroperitoneum $(30 \%-35 \%)$, and posterior mediastinum (20\%). Less common sites are the neck $(1 \%-5 \%)$ and pelvis $(2 \%-3 \%){ }^{2}$

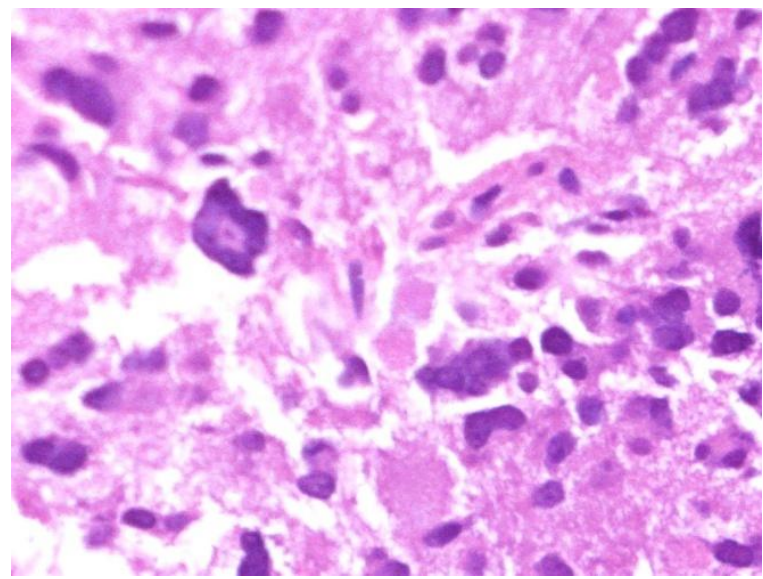

Fig 3: H\&E section shows tumor composed of ganglion cells in a background of spindled schwannian stroma, ganglion cells \& small cells consistent with ganglioneuroblastoma.

Patients with ganglioneuroblastoma most often present with pain (due to local effects from the primary tumor) and abdominal distention. At gross examination, ganglioneuroblastomas may be partially or totally encapsulated. Calcification is common, appearing discrete and punctuate as compared to neuroblastoma in which shows coarse and amorphous calcification. ${ }^{3}$ Histopathologically, they are malignant tumors containing primitive neuroblasts and mature ganglion cells. The patient presentation and prognosis depends on the amount of immature tissue present relative to the mature ganglion cells. Metastatic disease manifests radiographically as hepatomegaly, lucent submetaphyseal zones, periostitis, and cranial sutural widening (from dural metastases). Skeletal metastases (appearing as focal lucent or occasionally sclerotic areas) usually indicate towards more aggressive neuroblastoma. ${ }^{4}$

While the commonest age group for neuroblastoma is less than 1 year of age, the mass in our patient was present from the age of 8 months. So, the differential diagnosis of neuroblastoma had to be kept. Vascular encasement and compression can occur although invasion is very rare. Renal vessels may be sufficiently compressed to result in hypertension. ${ }^{3}$ Although vascular encasement has been described in ganglioneuroblastoma $\&$ neuroblastoma, such extensive encasement and displacement of vessels by a ganglioneuroblastoma has not been reported earlier.

Scintigraphic evaluation of ganglioneuroblastoma in a suspected case of neuroblastoma falls into two categories: identification of the primary tumor and metastatic surveillance. Identification of the primary tumor is performed with either a catecholamine analog (metaiodobenzylguanidine labeled to iodine123 , referred to as MIBG) or a somatostatin analog (pentetreotide labeled to indium-111). MIBG scintigraphy is highly sensitive (88\%) and specific (99\%) for sympathetic tissue such as those found in neuroblastoma, ganglioneuroblastoma, ganglioneuroma, carcinoid, and pheochromocytoma (but it 
does not enable discrimination among these tumors). ${ }^{5}$ Technetium-99m MDP scintigraphy is done for evaluation of bone disease. Tc-99m MDP scans are more sensitive than plain radiographs in the detection of bone metastases from neuroblastoma. ${ }^{6}$

Ultrasound provides most of the useful information about the size and nature of the mass lesion along with vessel encasement (especially in a child where high frequency linear probes can be used to screen the entire abdomen). However, contrast enhanced CT still scores over sonography to identify the exact nature and extent of the lesion, encasement/compression of vessels or displacement of other structures, lymphadenopathy and metastatic deposits. CT also helps to visualize the nature of calcification better than any other modality.

\section{Conclusion}

In a young child with abdominal mass, neurogenic tumors including neuroblastoma and variants like ganglioneuroblastoma are the commonest possibility. Such lesions show aggressive behaviour with extensive vascular encasement (as shown in this case), intraspinal extension, visceral (hepatic and splenic) metastases and skeletal metastases. These features can be well made out on imaging (sonography \& CT). Imaging also remains relevant during follow up of such lesions post chemotherapy $\&$ surgery.

\section{References}

1. Rha SE, Byun JY, Jung SE, Chon HJ, Lee HG, et. al. Neurogenic tumors in the abdomen: tumor types and imaging characteristics.RadioGraphics 2003; 23:2 9-43.

2. Morris JA, Shcochat SJ, Smith EI,Look, Brodeur GM, Cantor AB, et al. Biological variables in thoracic neuroblastoma: a Pediatric On-cology Group study. J Pediatr Surg 1995; 30:296-302, discussion 302-303.

3. Bousvaros A, Kirks DR, Grossman H. Imaging of neuroblastoma: an overview. Pediatr Radiol 1986; 16:89-106.

4. Abramson SJ. Adrenal neoplasms in children. Radiol Clin North Am 1997; 35:1415-1453.

5. Frappaz D, Giammarile F, Thiesse P, et al. False positive MIBG scan. Med Pediatr Oncol 1997; 29: 589-592.

6. Gilday DL, Ash JM, Reilly BJ. Radionuclide skeletal survey for pediatric neoplasms. Radiology 1977; 123:399-406. 